\title{
Small strokes in the carotid territory associated with a normal carotid arteriogram
}

\author{
PETER BRADSHAW AND JOHN GUMPERT \\ From the Department of Neurology of the United Sheffield Hospitals, Sheffield
}

SUMMARY Appropriate percutaneous carotid arteriography was carried out in 69 patients who presented with transient ischaemic attacks (TIAs) in the carotid territory. Major abnormalities were detected in 19 which included internal carotid stenosis (nine), internal carotid occlusion (seven), intracranial aneurysm (two), and cerebral angioma (one). Forty-five patients had normal angiograms and five slight irregularity of the internal carotid artery without stenosis. The symptoms and signs pertaining to the normotensive and hypertensive groups are presented. The outcome of stroke in 35 normotensives was as follows: three died of related disease, three are severely disabled, two have slight disability, eight have minor neurological residua, and 19 are normal. Fifteen patients had a diastolic blood pressure of above $105 \mathrm{~mm} \mathrm{Hg}$ and the outcome of stroke in these patients treated with hypotensive agents was as follows: two died of related disease, two have severe and two moderate disablement, four have slight disability, one has minor residua, and four are normal. The pathogenesis of TIAs in relation to the two groups and the use of anticoagulant drugs and hypo= tensive agents are discussed.

During a previous study of stroke associated with occlusive carotid artery disease (Bradshaw and Casey, 1967), other patients were seen who presented with transient ischaemic attacks (TIAs) and who had normal carotid angiograms. This paper is based on a clinical study of 50 such patients and its purpose is to present information concerning pathogenesis, prognosis, and treatment of this syndrome.

The introduction of carotid angiography by Moniz, Lima, and Lacerda (1937) led to the recognition of occlusive disease involving the great vessels in the neck as a cause of stroke. The different modes of stroke presentation associated with stenosis or occlusion of the internal carotid artery have been described by Fisher (1951), Johnson and Walker (1951), Smythe (1954), Symonds (1957), Marshall (1960), and others. These include 'sudden stroke', in which lasting disability reaches maximum severity within eight hours; 'ingravescent stroke', when disability increases for more than eight hours; and 'transient ischaemic attacks', in which disability lasts for between minutes and 24 hours. When neurological residua persist for more than 24 hours, some (Marshall, 1968) prefer the designation 'incomplete sudden stroke'. However, wherp more than one TIA has occurred before initia 3 assessment, it may be impossible to say whethes. the first or a subsequent episode gave rise to persistent neurological signs. For this reason we prefer to include as TIAs both those which resolve completely within 24 hours and others in which there is substantial recovery but some minor neurological residua remain.

Only a proportion of patients presenting with TIAs in the territory of the carotid artery have occlusive disease of the internal carotid or parent arteries. Bull, Marshall, and Shaw (1960) submitted 79 patients, who had sustained various forms of stroke, to successful appropriate single vessel angiography and in 45 radiographs were normal. Burrows and Marshall (1965) used comprehensive four vessel angiography in a similar series and, although angiographic abnormalities were detected in $67 \%$, these were not always sited appropriately to explain the neurological deficit. Marshall (1968) has shown that when intracranial haemorrhage can be excluded by lumbar puncture and echoencephalography, 
normal angiographs are most likely to be associated with stroke in the patient who exhibits a diastolic blood pressure above $105 \mathrm{~mm} \mathrm{Hg}$.

Most are agreed that the prognosis for TIAs in the carotid artery territory is grave. The proportion who later become disabled by massive stroke has been reported to be between 50 and $70 \%$ (Acheson and Hutchinson, 1964; Marshall, 1964; Bradshaw and Casey, 1967). Further, Marshall has pointed out that disablement is unrelated to the number of TIAs and, in $60 \%$ of those disabled, the cerebrovascular catastrophe ensues within two months of the onset. However, others, including Hurwitz, Groch, Wright, and McDowell (1959) and Friedman, Wilson, Mosier, Colandrea, and Nichaman (1969) have reported a more benign prognosis. The disagreement might be explained if there were a difference in prognosis between those with and those without occlusive disease of the appropriate internal carotid artery.

\section{METHODS}

In April 1965 a prospective study was commenced of patients admitted to the Department of Neurology of the United Sheffield Hospitals under the personal care of one of us (P.B.), who had had one or more TIA in the territory of the internal carotid artery. Patients over the age of 64 years and two others who displayed evidence of gross peripheral arterial disease were excluded. Care was taken also to exclude patients with neurological migraine according to the criteria described by Bradshaw and Parsons (1965). By TIA is here understood a disturbance of function attributable to vascular insufficiency within the territory supplied by the internal carotid artery which resolves either completely or very substantially within the space of 24 hours. Three patients were accepted who exhibited homonymous hemianopia because in all there was other evidence, such as dysphasia or hemiplegia, to suggest that the carotid rather than the vertebrobasilar circulation was affected.

Percutaneous carotid arteriography was carried out on all patients and the series was closed when $\mathbf{5 0}$ patients with normal or near normal angiograms had been accepted. Seven of the first nine patients also had an arch aortogram. Routine investigation included the following: full blood count and erythrocyte sedimentation rate (ESR), urinalysis, plain radiographs of chest and skull, examination of the cerebrospinal fluid (CSF) and at least one electrocardiogram (ECG). Blood pressures were recorded six hourly for the first five days and when a diastolic pressure of $105 \mathrm{~mm} \mathrm{Hg}$ or over was sustained the patient was deemed to be hypertensive. All hypertensive patients were further investigated by estimation of the plasma urea, electrolytes, creatinine clearance, and an intravenous pyelogram.

Details of treatment are included under the appropriate section. All patients were followed personally and designated as follows:

1. Normal-neither symptoms nor signs.

2. Minor residua-asymptomatic, no more than slight reflex asymmetry and/or a feebly extensor plantar response.

3. Slight disability - one or more of the following: homonymous hemianopia, relatively slight weakness or clumsiness of a limb, slight sensory change but able to travel on public transport and to perform full-time light work.

A final designation of any of the above was considered satisfactory from the neurological viewpoint.

4. Moderate disablement-one or more of the following: slight dysphasia, moderate spasticity and weakness with or without cutaneous sensory loss, able to walk 500 yards with either a toe-raising spring and/or a stick.

5. Severe disablement - any disability greater than the above.

All but nine patients were observed throughout the period of follow-up. Of the remainder, it was discovered that three had died and details were obtained from the family doctor. The remainder were seen personally shortly before the conclusion of the series.

\section{RESULTS}

Appropriate percutaneous carotid arteriography was carried out on 69 patients who presented with transient ischaemic attacks. Abnormalities were detected in 19 which included internal carotid stenosis (nine), internal carotid occlusion (seven), aneurysm of the posterior communicating artery (two), and cerebral angioma (one). In the last three mentioned, the lumbar cerebrospinal fluid (CSF) was normal and the appearances of the intracranial circulation did not indicate any space occupying lesion. In five other patients, there was a slight irregularity involving the internal carotid artery throughout the greater part of its course in the neck, but without stenosis. In 45 patients the radiographs were normal. Seven of the first nine patients, with entirely normal carotid asteriograms, also had an arch aortogram: good filling of all relevant vessels in the thorax and neck was obtained and the appearances were normal.

The 50 patients with normal or near normal carotid arteriograms comprise the present study. Fifteen had a diastolic blood pressure of $105 \mathrm{~mm} \mathrm{Hg}$ or above and were labelled hypertensive; the remainder 
comprised six patients with diastolic pressures of 100 to $104 \mathrm{~mm} \mathrm{Hg}$ which settled on rest and sedation and 29 in whom diastolic pressures remained throughout below $100 \mathrm{~mm} \mathrm{Hg}$. These were labelled normotensive.

A history indicative of previous extracranial vascular disorder was obtained in two normotensives; one who had sustained a myocardial infarct four years previously and one who had experienced angina pectoris for the past two years. One hypertensive patient gave a family history of siblings who died before the fifth decade of presumed vascular cause. No patient admitted to symptoms that resembled neurological migraine. In both hypertensive and normotensive subjects the ratio of men to women was 12:1, and the average age of onset was 51 years (26 to 63 ).

The interval between the first TIA and initial assessment averaged 14 days (five to 27). The incidence of TIAs before admission is shown in Table 1 and the duration of the first or only TIA is shown in Table 2. In 17 of 23 who had more than one attack, subsequent episodes tended to become more pro-

TABLE 1

NUMBER OF TIAS BEFORE FIRST ASSESSMENT

\begin{tabular}{lccc}
\hline & Normotensive & Hypertensive & Total \\
\hline Single & 23 & 4 & 27 \\
$2-3$ & 7 & 9 & 16 \\
$4-5$ & 3 & 2 & 5 \\
Multiple & 2 & 0 & 2 \\
\hline & 35 & 15 & 5 \\
\hline
\end{tabular}

TABLE 2

DURATION OF FIRST OR ONLY TIA

\begin{tabular}{lccc}
\hline Hours & Normotensive & Hypertensive & Total \\
\hline Less than 1 & 12 & 7 & 19 \\
$1-2$ & 12 & 1 & 13 \\
$2-3$ & 8 & 6 & 14 \\
$3-9$ & 3 & 1 & 4 \\
\hline & 35 & 15 & 50 \\
\hline
\end{tabular}

found, more extensive, or longer lasting. Insufficieney involved the distribution of the right internal carotid artery in 27 and the left in 33, though in two of those who were hypertensive there was bilateral involvement in the first attack. The distribution of symptoms is shown in Table 3. The pattern of the TIAs was as follows.
TABLE 3

SYMPTOMS EXPERIENCED DURING TIAS

\begin{tabular}{lrrr}
\hline & Normotensive & Hypertensive & Both \\
\hline Dysphasia & 15 & 6 & 21 \\
Monocular blindness & 3 & 15 & 34 \\
Weakness total & 29 & 3 & 11 \\
face & 8 & 14 & 40 \\
upper limb & 26 & 5 & 16 \\
lower limb & 11 & 12 & 26 \\
Paraesthesiae total & 14 & 5 & 12 \\
face & 7 & 11 & 23 \\
upper limb & 12 & 2 & 4 \\
lower limb & 2 & & \\
\hline
\end{tabular}

Forty-six patients experienced either weakness or paraesthesiae or both referred to the face (11), the upper limb (31), or the lower limb (26). Twenty-one complained of speech disturbance which was probably dysphasia; in two of these this was an isolated abnormality, in 19 there were also other symptoms. Three patients noticed monocular blindness which was the only symptom in two and associated with other abnormalities in one.

The distribution of physical signs is shown in Table 4. Three normotensive patients had a bruit $\vec{\circ}$

TABLE 4

PHYSICAL SIGNS

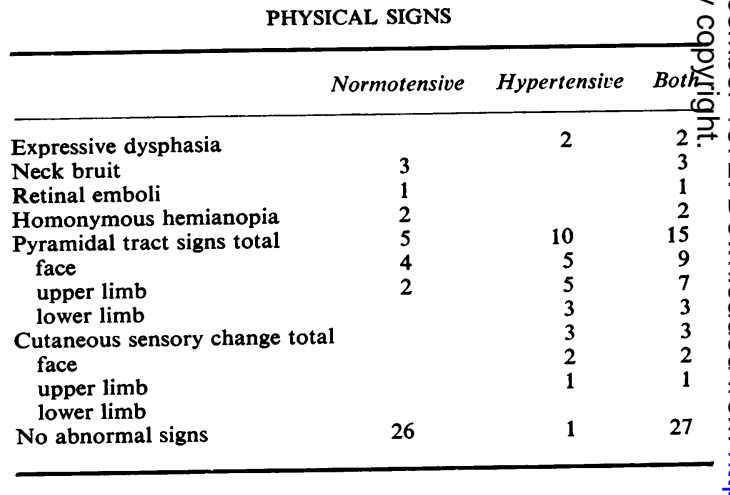

over the appropriate side of the neck and one had ipsilateral retinal emboli. Irregularity of the calibre of the retinal arterioles was not seen nor was there clinical evidence of peripheral arterial disease. Neuro- $\delta$ logical signs in normotensive patients were infrequent $₹$ and slight in extent. Though two had homonymous $ᄋ$ hemianopia, both had experienced symptoms to sug- $>$ gest that the carotid rather than the vertebrobasilaro circulation was involved. Twenty-six of the 35 normotensive patients were normal. In contrast, moderate? 
signs referable to the pyramidal or long sensory tracts were detected in the majority of hypertensive patients of whom only one was normal.

INVESTIGATIONS Routine investigations were unremarkable with the exception of the following. Mild iron deficiency anaemia with haemoglobin levels of 70 and $75 \%$ respectively was detected in two patients. Two others had a transient increase in the polymorphonuclear leucocytes with total white cell counts of 12,000 and 11,000 per c.mm respectively. The electrocardiogram (ECG) showed evidence of a recent posterior myocardial infarction in one, an old myocardial infarct in one, and atrial fibrillation in one other. Left axis deviation was seen in one normotensive and six hypertensive patients. Plain radiographs of the chest revealed slight cardiac enlargement in five hypertensive and in one normotensive patient. Plain radiographs of the skull showed slight calcification in the vicinity of the internal carotid siphon in two. Urinalysis and examination of the lumbar CSF were normal in all 50 patients.

Intravenous pyelography and creatinine clearance were carried out on 15 hypertensive patients and results were normal. An electroencephalogram (EEG) was done on six patients and was normal in five; one displayed low voltage slow wave activity over the appropriate cerebral hemisphere which was less marked when the record was repeated three weeks later.

TREATMENT Nine of the 35 normotensive patients were treated with anticoagulants. These included one with ECG evidence of a recent myocardial infarction, one with atrial fibrillation, five in whom arteriography suggested slight diffuse atheroma of the internal carotid artery and two who continued to experience multiple TIAs. All were given warfarin, and prothrombin times of two to three times that of fresh controls were obtained in hospital. Prothrombin times were measured at intervals of between one and four weeks thereafter as seemed fitting and treatment was maintained for 12 to 18 months. In those patients followed for longer periods, the dose of warfarin was slowly reduced and discontinued after four to six weeks. No major complications of such treatment were encountered. Six other patients in whom diastolic blood pressures of 95 to 104 $\mathrm{mm} \mathrm{Hg}$ were recorded were treated with diazepam, 2 to $5 \mathrm{mg}$ eight-hourly, and, in all, diastolic pressures fell and remained below $95 \mathrm{~mm} \mathrm{Hg}$ during the period of observation. One normotensive patient with atrial fibrillation was treated with digoxin and two with iron deficiency anaemia received ferrous fumerate tablets for two months when haemoglobin levels were normal.
All 15 hypertensive patients were initially treated with methyl dopa, 250 to $500 \mathrm{mg}$ eight-hourly. In 10 patients diastolic pressures of 90 to $100 \mathrm{~mm} \mathrm{Hg}$ measured in the erect posture were maintained, but in five, adequate control of blood pressure was not obtained and medication was changed to bethanidine 10 to $30 \mathrm{mg}$. This achieved diastolic pressures of less than $95 \mathrm{~mm} \mathrm{Hg}$.

\section{PROGRESS}

Thirty-five normotensive patients were followed for between nine months and six years. The duration of follow-up and whether the final assessment was deemed satisfactory or unsatisfactory are shown in Fig. 1. One patient died within 19 months of unverified cerebral haemorrhage, one succumbed to unverified sudden stroke associated with myocardial infarction after two years and 10 months, during which time he was moderately disabled, and one died of verified thrombosis of the contralateral internal carotid within the siphon 14 months after the first TIA. Final assessment of the 32 survivors was as follows: three became severely disabled by sudden stroke all within four months of their first TIA, two have slight disability, eight display minor neurological residua, and 19 are normal. No patient who received anticoagulants became disabled or died. Thus the outcome of TIAs in normotensive patients was satisfactory in $80 \%$.

The 15 hypertensive patients were followed for between 11 months and six years. The duration of the follow-up and the results of final assessment are shown in Fig. 2. Two patients died of unverified cerebral haemorrhage after intervals of 10 months, and two years and six months respectively. Four patients sustained sudden strokes, two of whom are severely disabled and two have moderate disability. These were all readmitted to the neurological department within 24 hours of stroke and in each the diastolic blood pressure was above $105 \mathrm{~mm} \mathrm{Hg}$. Ultrasound echography showed no displacement of intracranial contents and the lumbar CSF was normal; further angiography was not carried out. These sudden strokes ensued six months to three years after the first TIA. Three other patients have slight disability, two display minor neurological residua, and four are normal. Thus the outcome of hypertensive TIAs was satisfactory in $60 \%$. It proved possible to maintain 


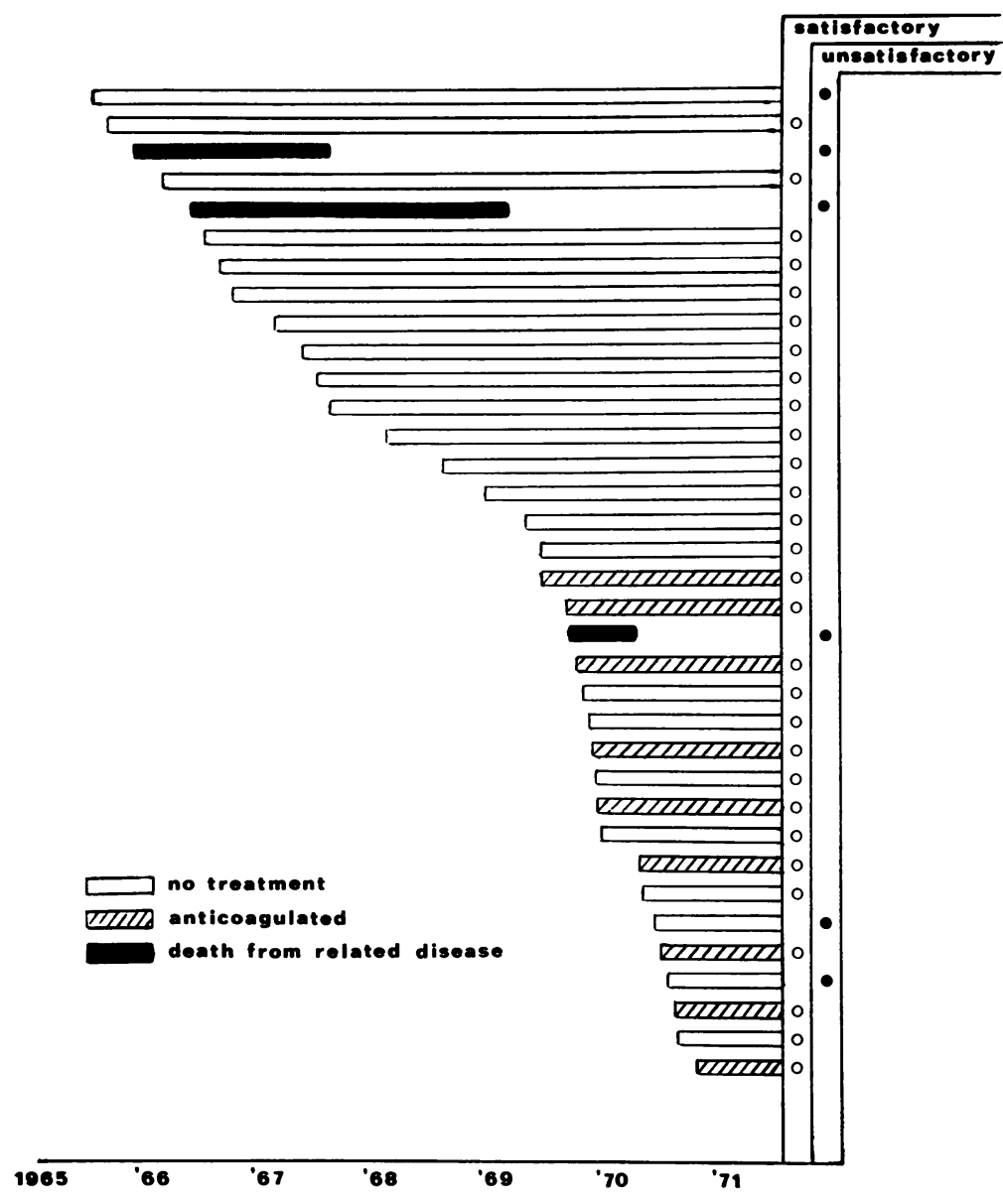

FIG. 1. Normotensive patients. Duration of follow-up and final assessment of 35 normotensive patients.

diastolic blood pressure below $100 \mathrm{~mm} \mathrm{Hg}$ in eight of the nine patients who fared well but in one who suffered from a chronic anxiety state diastolic pressures varied between 90 and 110 $\mathrm{mm} \mathrm{Hg}$ throughout the period of observation.

\section{DISCUSSION AND CONCLUSIONS}

Sixty-nine patients under the age of 64 years, who presented with one or more TIA in the territory of the internal carotid artery were submitted to appropriate single vessel angiography and major abnormalities were detected in 19 . Abnormalities included internal carotid stenosis (nine), internal carotid occlusion (seven), intracranial aneurysm (two), and cerebral angioma (one). In the last three mentioned, the lumbar CSF was normal, the angiographic appearances of the intracranial circulation were unremarkable, and there was no clinical feature to suggest intracranial bleeding. These observations stress the importance of investigating normotensive TIAs by angiography, especially if the use of anticoagulant drugs is contemplated. Our figures suggest that about one-third of patients presenting in this fashion have major arteriographic 8 abnormalities. In 50 patients single vessel angiography was essentially normal, though in five of 웅 these there was a slight irregularity of the lumen of the internal carotid artery suggestive of atheroma without stenosis. Fifteen of the $50 \mathrm{~N}$ patients had a diastolic blood pressure above 


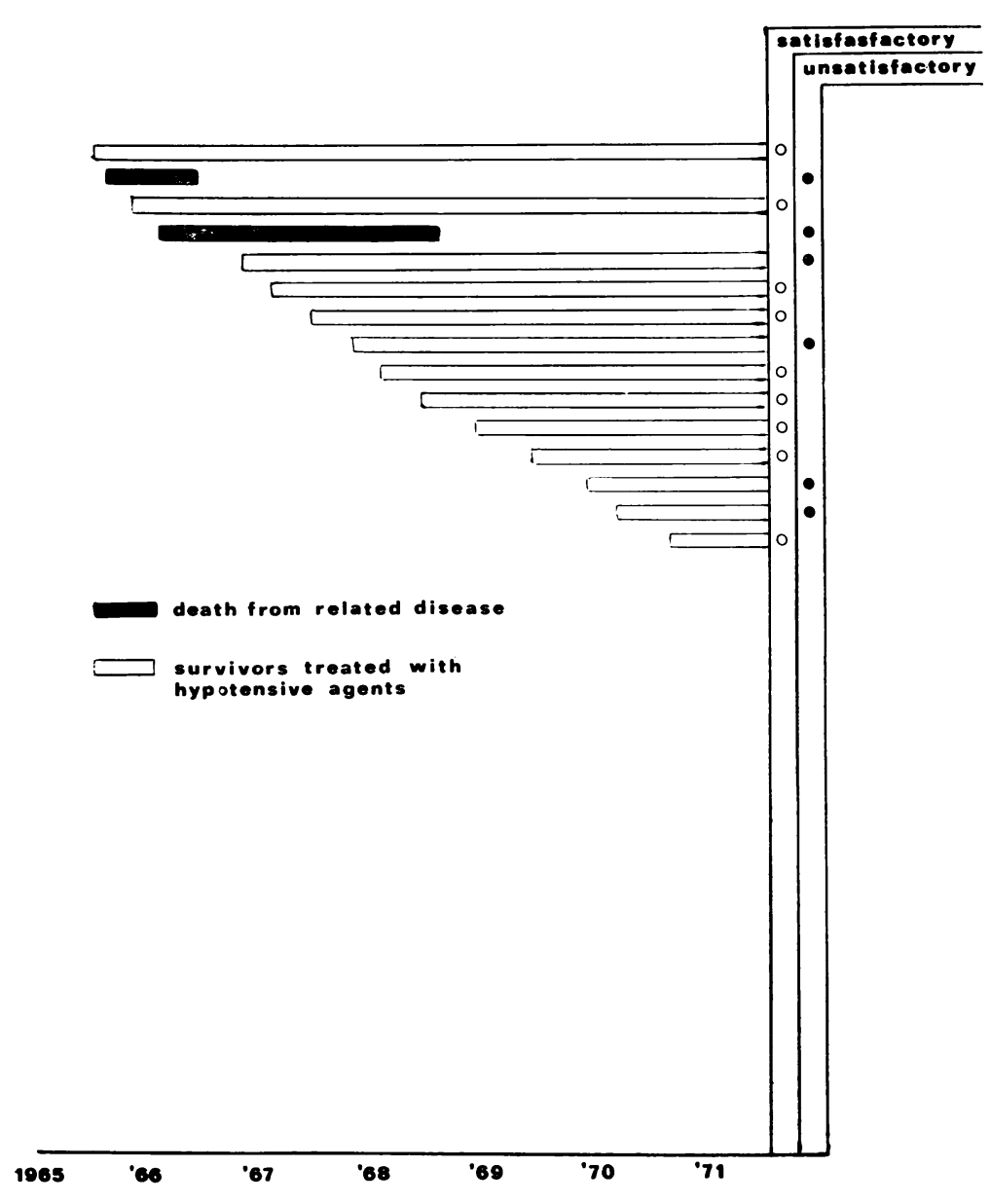

FIG. 2. Hypertensive patients.

Duration of follow-up and final assessment of 15 hypertensive patients. Twelve patients attended follow-up clinics at intervals of months throughout; three failed to keep appointments. Postal inquiries from relatives and family doctors suggested that two of the defaulters had died of myocardial infarction. The third was seen in his home shortly before the study was completed. At no time during attendances at clinics was there any evidence that blood pressure had risen above 100 mm Hg. Despite this, four patients were readmitted as emergencies at the request of their doctors having sustained further sudden strokes and when examined were found to have diastolic pressures over $105 \mathrm{~mm} \mathrm{Hg}$. These second strokes occurred after intervals of between six months and $2 \frac{1}{2}$ years from first assessment. The resultant disability was moderate in two and severe in two. All strokes occurred upon the same side as the initial TIA and none made sufficient recovery to be able to return to work. Repeat angiography was not carried out but the lumbar CSF was free from blood and echograms showed no displacement of the third ventricle.

$104 \mathrm{~mm} \mathrm{Hg}$ and these were labelled hypertensive. This proportion probably underestimates the incidence of hypertension in this syndrome, as it is likely that hypertensive patients would be referred more frequently to a general physician than to a neurologist. Seven of the first nine patients with entirely normal angiograms, including four normotensive and three hypertensive patients, also had a normal arch aortogram. Thus it is improbable that any significant proportion of the remainder had occlusive arterial disease proximal to the site in the common carotid artery where contrast material had been injected.

The ratio of men to women was $12: 1$, the average age of onset was 51 years in both normotensive and hypertensive groups and only two had evidence of extracranial atheroma. Bradshaw and Casey (1967) reported a similar series of patients in whom TIAs were associated with stenosis or occlusion of the internal carotid artery. In this former series, the ratio of men to women was $3: 1$, the average age at presentation was 55 years, and many of the patients had clinical evidence of peripheral vascular disease. Between the two series there is no essential difference in the pattern of TIAs; in both series the majority of those patients who had more than one attack noted that subsequent episodes became more extensive, more profound, or longer lasting. Although, in the present study, few of the normotensive patients had significant neurological signs, in both series monocular blindness was a symptom, homonymous hemi- 
anopia was detected, and a bruit over the appropriate side of the neck heard. It is difficult to escape the conclusion that TIAs associated with normal single vessel angiograms represent an earlier stage in the same pathological process that may later give rise to stenosis or occlusion of the internal carotid artery. Indeed, one of our patients later succumbed to verified carotid occlusion on the opposite side.

The pathogenesis of normotensive TIAs associated with normal carotid angiography remains undetermined but there is much to suggest that a proportion owe their attacks to cerebral embolism. In two patients, one with a recent myocardial infarct and the other with atrial fibrillation, embolism was presumed to have occurred. However, it is seldom that a cardiac source of emboli can be identified. In one patient, retinal emboli were observed and in two others who experienced multiple TIAs, these ceased after administration of warfarin and have not recurred. The appearances at angiograply in five suggested that atheroma of the internal carotid might be a reservoir of emboli. Wolman and Bradshaw (1967) described embolic occlusion of segmental branches of the aorta by atheromatous material as a cause of ischaemic myelopathy and it seems likely that similar emboli may affect the cerebral circulation arising from atheromatous ulceration of the great vessels in the thorax and neck which might not be revealed by angiography. We have little to add to our understanding of the cause of TIAs in hypertensive patients, except to say that the high incidence of persistent physical signs favours small haemorrhages from miliary aneurysms described by Charcot and Bouchard (1868) over 100 years ago.

The prognosis for TIAs in normotensive patients is favourable in $80 \%$ when single vessel angiography is normal. Although three patients died of related disorder and three became severely disabled by sudden stroke within four months of their first TIA the outcome is far more favourable than obtains in occlusive carotid disease in which, as Marshall (1968) has shown, some $70 \%$ become disabled, and of these the majority have sudden stroke within two months of the onset. The relatively benign prognosis makes it impossible to assess the value of anticoagulant treatment and a control trial will be necessary. However, none of our patients who were so treated either died or became disabled. The prognosis for hypertensive TIAs is more grave, despite every effort to keep the diastolic blood pressure below $100 \mathrm{~mm} \mathrm{Hg}$. Two died of related disease and four others became disabled. All of these four were found to have a rise of diastolic blood pressure to above 105 $\mathrm{mm} \mathrm{Hg}$ within 24 hours of sudden stroke. Out of nine patients who fared well, it was possible to keep diastolic blood pressures below $100 \mathrm{~mm} \mathrm{Hg}$ in eight. Nevertheless, a satisfactory outcome was obtained in $60 \%$.

Acheson and Hutchinson (1971) in a review of various forms of stroke noted that the prognosis for focal attacks of cerebrovascular insufficiency was more grave in the presence of hypertension and in others who displayed ECG abnormalities. Their paper, however, does not correlate the prognosis with angiographic findings and it is of interest to find that, when there is no radio- $c$ graphic abnormality as in the present study, the of connection between a poor prognosis and $\infty$ hypertension remains valid. Since only 10 of ou黑 $\overrightarrow{0}$ patients had ECG abnormalities, we are unable to confirm or disprove the relevance to prognosis

Our thanks are due to Dr. Ronald Grainger and Dr Norman Lewtas who carried out and reported the results of neuroradiological studies.

\section{ADDENDUM}

Since this study was completed, Marshall and Wilkinson (1971) have published a similar series which shows a significantly higher incidence of cardiac lesions. Furthermore, in similar studies associated with occlusive disease of the carotid artery, Bradshaw and Casey (1967) reported a favourable outcome in about two-thirds of their cases, whereas Marshall (1960) reported a favourable outcome in one-third of his patients. We suggest that these discrepancies may be related to the method of selection of patients between the series. We would mention however, that Barham Carter (1971), (personal communication) has commented that his figures are very similar to ours.

\section{REFERENCES}

Acheson, J., and Hutchinson, E. C. (1964). Observations on the natural history of transient cerebral ischaemia. Lancet, 2, 871-874. 
Acheson, J., and Hutchinson, E. C. (1971). The natural history of 'focal cerebral vascular disease'. Quarterly Journal of Medicine, 40, 15-23.

Barham Carter (1971). Personal communication.

Bradshaw, P., and Casey, E. (1967). Outcome of medically treated stroke associated with stenosis or occlusion of the internal carotid artery. British Medical Journal, 1, 201-205.

Bradshaw, P., and Parsons, M. (1965). Hemiplegic migraine, a clinical study. Quarterly Journal of Medicine, 34, 65-85.

Bull, J. W. D., Marshall, J., and Shaw, D. A. (1960). Cerebral angiography in the diagnosis of the acute stroke. Lancet, 1 , 562-565.

Burrows, E. H., and Marshall, J. (1965). Angiographic investigation of patients with transient ischaemic attacks. Journal of Neurology, Neurosurgery, and Psychiatry, 28, 533-539.

Charcot, J.-M., and Bouchard, C. (1868). Nouvelles recherches sur la pathogénie de l'hémorrhagie cérébrale. Archives de Physiologie Normale et Pathologique, 1, 110127.

Fisher, M. (1951). Occlusion of the internal carotid artery. Archives of Neurology and Psychiatry, 65, 346-377.

Friedman, G. D., Wilson, W. S., Mosier, J. M., Colandrea, M. A., and Nichaman, M. Z. (1969). Transient ischaemic attacks in a community. Journal of the American Medical Association, 210, 1428-1434.

Hurwitz, L. J., Groch, S. N., Wright, I. S., and McDowell, F. H. (1959). Carotid artery occlusive syndrome. Archives of Neurology, 1, 491-501.
Johnson, H. C., and Walker, A. E. (1951). The angiographic diagnosis of spontaneous thrombosis of the internal and common carotid arteries. Journal of Neurosurgery, 8, 631659.

Marshall, J. (1960). Carotid insufficiency. Transactions of the Ophthalmological Society of the United Kingdom, 80, 263265.

Marshall, J. (1964). The natural history of transient ischaemic cerebrovascular attacks. Quarterly Journal of Medicine, 33, 309-324.

Marshall, J. (1968). The Management of Cerebrovascular Disease. 2nd edn. Churchill: London.

Marshall, J., and Wilkinson, I. M. S. (1971). The prognosis of carotid transient ischaemic attacks in patients with normal angiograms. Brain, 94, 395-402.

Moniz, E., Lima, A., and Lacerda, R. de (1937). Hémiplégies par thrombose de la carotide interne. Presse Médicale, 45, I, 977-980.

Smyth, G. E. (1954). Clinical aspect of carotid thrombosis. Proceedings of the Royal Society of Medicine, 47, 602.

Symonds, Sir C. (1957). Occlusion of the internal carotid arteries. Modern Trends in Neurology. 2nd series, pp. 91-104. Edited by D. Williams. Butterworth: London.

Wolman, L., and Bradshaw, P. (1967). Spinal cord embolism. Journal of Neurology, Neurosurgery, and Psychiatry, 30, 446-454. 